\title{
Improved Estimators of Population Mean Using Two Auxiliary Variables in Stratified Random Sampling
}

\author{
Rajesh Singh \\ Department of Statistics \\ Banaras Hindu University (U.P.), India \\ rsinghstat@yahoo.com \\ Mukesh Kumar \\ Department of Statistics \\ Banaras Hindu University (U.P.), India \\ mukesh.stat@gmail.com
}

\begin{abstract}
An exponential family of estimators, which use the information of two auxiliary variables in the stratified sampling, is proposed to estimate the population mean of the variable under study. The mean-squared error of the suggested family of estimators are derived under large sample approximation. The family of estimators in its optimum case is carried out to show the properties of the proposed estimators.
\end{abstract}

Keywords: Auxiliary information, Study variate, Stratified random sampling, Mean square error, Exponential estimator.

\section{Introduction}

The problem of estimating the population mean in the presence of an auxiliary variable has been widely discussed in finite population sampling literature. Out of many ratio, product and regression methods of estimation are good examples in this context. Diana (1993) suggested a class of estimators of the population mean using one auxiliary variable in the stratified random sampling and examined the mean square Error (MSE) of the estimators up to the $k^{\text {th }}$ order of approximation. Kadilar and Cingi (2003), Singh, H.P. et. al. (2008), Singh and Vishwakarma (2008), Singh, R.et al. (2008), Koyuncu and Kadilar (2009) proposed estimators in stratified random sampling. Singh (1967) and Perri (2007) suggested some ratio cum product estimators in simple random sampling. Bahl and Tuteja (1991) and Singh, R. et. al. (2008, 2009) suggested some exponential ratio type estimators. Ghosh (1958) and Rao (1977) have suggested estimators in stratified random sampling with multiple characteristics. In this study, we suggest some exponential-type estimators using the auxiliary information in the stratified random sampling when two auxiliary variables are available.

Consider a finite population $U=\left(U_{1}, U_{2}, U_{3} \ldots U_{N}\right)$ of size $N$ and let $y, x$ and $z$, respectively, be the study and two auxiliary variables associated with each $\mathrm{Uj}$ $(j=1,2,3 . . N)$ of the population. Let the population of size, $N$, is stratified into $L$ strata with $h^{\text {th }}$ stratum containing $N_{h}$ units, where $h=1,2,3, \ldots L$ such that $\sum_{h=1}^{L} N_{h}=$ $\mathrm{N}$. 
A simple random sample of size $n_{h}$ is drawn without replacement from the $h_{\text {th }}$ stratum such that $\sum_{\mathrm{h}=1}^{\mathrm{L}} \mathrm{n}_{\mathrm{h}}=\mathrm{n}$. Let $\left(\mathrm{y}_{\mathrm{hi}}, \mathrm{x}_{\mathrm{hi}}, \mathrm{z}_{\mathrm{hi}}\right)$ denote the observed values of $\mathrm{y}, \mathrm{x}$, and $z$ on the $i_{\text {th }}$ unit of the $h_{\text {th }}$ stratum, where $i=1,2,3 . . N_{h}$.

To obtain the bias and MSE, we write

$\overline{\mathrm{y}}_{\mathrm{st}}=\sum_{\mathrm{h}=1}^{\mathrm{L}} \mathrm{w}_{\mathrm{h}}^{\prime} \overline{\mathrm{y}}_{\mathrm{h}}=\overline{\mathrm{Y}}\left(1+\mathrm{e}_{0}\right), \quad \overline{\mathrm{x}}_{\mathrm{st}}=\sum_{\mathrm{h}=1}^{\mathrm{L}} \mathrm{w}_{\mathrm{h}} \overline{\mathrm{x}}_{\mathrm{h}}=\overline{\mathrm{X}}\left(1+\mathrm{e}_{1}\right)$

$\overline{\mathrm{z}}_{\mathrm{st}}=\sum_{\mathrm{h}=1}^{\mathrm{L}} \mathrm{w}_{\mathrm{h}}^{\prime} \overline{\mathrm{Z}}_{\mathrm{h}}=\overline{\mathrm{Z}}\left(1+\mathrm{e}_{2}\right)$.

Such that,

$\mathrm{E}\left(\mathrm{e}_{0}\right)=\mathrm{E}\left(\mathrm{e}_{1}\right)=\mathrm{E}\left(\mathrm{e}_{2}\right)=0$.

$V_{r s t}^{r}=\sum_{h=1}^{L} W_{h}^{r+s+t} \frac{E\left[\left(\bar{y}_{h}-\overline{\mathrm{Y}}_{\mathrm{h}}\right)^{\mathrm{r}}\left(\overline{\mathrm{x}}_{\mathrm{h}}-\overline{\mathrm{X}}_{\mathrm{h}}\right)^{\mathrm{s}}\left(\overline{\mathrm{z}}_{\mathrm{h}}-\overline{\mathrm{Z}}_{\mathrm{h}}\right)^{\mathrm{t}}\right]}{\overline{\mathrm{Y}}^{\mathrm{r}} \overline{\mathrm{X}}^{\mathrm{s}} \overline{\mathrm{Z}}^{\mathrm{t}}}$

where,

and

$$
\begin{aligned}
& \left.\left.\overline{\mathrm{y}}_{\mathrm{st}}=\sum_{\mathrm{h}=1}^{\mathrm{L}} \mathrm{w}_{\mathrm{h}} \overline{\mathrm{y}}_{\mathrm{h}}, \quad \overline{\mathrm{y}}_{\mathrm{h}}=\frac{1}{\mathrm{n}_{\mathrm{h}}}\right\rangle_{\mathrm{i}=1}^{\mathrm{n}_{\mathrm{h}}} \mathrm{y}_{\mathrm{hi}}, \quad \overline{\mathrm{Y}}_{\mathrm{h}}=\frac{1}{\mathrm{~N}_{\mathrm{h}}}\right\rangle_{\mathrm{i}=1}^{\mathrm{n}_{\mathrm{h}}} \mathrm{Y}_{\mathrm{hi}}, \\
& Y=\overline{\mathrm{Y}}_{\mathrm{st}}=\sum_{\mathrm{h}=1} \mathrm{w}_{\mathrm{h}} \overline{\mathrm{Y}}_{\mathrm{h}} \mathrm{w}_{\mathrm{h}}=\frac{\mathrm{N}_{\mathrm{h}}}{\mathrm{N}},
\end{aligned}
$$

$\mathrm{V}\left(\overline{\mathrm{y}}_{\mathrm{st}}\right)=\overline{\mathrm{Y}}^{2} \mathrm{~V}_{200}^{*}$

Similar expressions for $X$ and $Z$ can also be defined.

Using (1.1), we can write

where

$$
\begin{aligned}
\mathrm{E}\left(\mathrm{e}_{0}^{2}\right)=\frac{\sum_{\mathrm{h}=1}^{\mathrm{L}} \mathrm{w}_{\mathrm{h}}^{2} \gamma_{\mathrm{h}} \mathrm{S}_{\mathrm{yh}}^{2}}{\overline{\mathrm{Y}}^{2}}=\mathrm{V}_{200}, & \mathrm{E}\left(\mathrm{e}_{1}^{2}\right)=\frac{\sum_{\mathrm{h}=1}^{\mathrm{L}} \mathrm{w}_{\mathrm{h}}^{2} \gamma_{\mathrm{h}} \mathrm{S}_{\mathrm{xh}}^{2}}{\overline{\mathrm{X}}^{2}}=\mathrm{V}_{020}^{r}, \\
\mathrm{E}\left(\mathrm{e}_{2}^{2}\right)=\frac{\sum_{\mathrm{h}=1}^{\mathrm{L}} \mathrm{w}_{\mathrm{h}}^{2} \gamma_{\mathrm{h}} \mathrm{S}_{\mathrm{zh}}^{2}}{\overline{\mathrm{Z}}^{2}}=\mathrm{V}_{002}, & \mathrm{E}\left(\mathrm{e}_{0} \mathrm{e}_{1}\right)=\frac{\sum_{\mathrm{h}=1}^{\mathrm{L}} \mathrm{w}_{\mathrm{h}}^{2} \gamma_{\mathrm{h}} \mathrm{S}_{\mathrm{xyh}}}{\overline{\mathrm{XY}}}=\mathrm{V}_{110}^{r}, \\
\mathrm{E}\left(\mathrm{e}_{1} \mathrm{e}_{2}\right)=\frac{\sum_{\mathrm{h}=1}^{\mathrm{L}} \mathrm{w}_{\mathrm{h}}^{2} \gamma_{\mathrm{h}} \mathrm{S}_{\mathrm{Xzh}}}{\overline{\mathrm{X} \overline{\mathrm{Z}}}}=\mathrm{V}_{011}, & \mathrm{E}\left(\mathrm{e}_{0} \mathrm{e}_{2}\right)=\frac{\sum_{\mathrm{h}=1}^{\mathrm{L}} \mathrm{w}_{\mathrm{h}}^{2} \gamma_{\mathrm{h}} \mathrm{S}_{\mathrm{Xzh}}}{\overline{\overline{\mathrm{X}} \overline{\mathrm{Z}}}}=\mathrm{V}_{101}^{r},
\end{aligned}
$$

$$
\begin{gathered}
S_{y h}^{2}=\frac{\sum_{i=1}^{N_{h}}\left(\bar{y}_{h}-\bar{Y}_{h}\right)^{2}}{N_{h}-1}, \quad S_{x h}^{2}=\frac{\sum_{i=1}^{N_{h}}\left(\bar{x}_{h}-\bar{X}_{h}\right)^{2}}{N_{h}-1}, \\
S_{z h}^{2}=\frac{\sum_{i=1}^{N_{h}}\left(\bar{z}_{h}-\bar{Z}_{h}\right)^{2}}{N_{h}-1}, \quad S_{x y h}=\frac{\sum_{i=1}^{N_{h}}\left(\bar{x}_{h}-\bar{X}_{h}\right)\left(\bar{y}_{h}-\bar{Y}_{h}\right)}{N_{h}-1}, \\
S_{y z h}=\frac{\sum_{i=1}^{N_{h}}\left(\bar{y}_{h}-\bar{Y}_{h}\right)\left(\bar{z}_{h}-\bar{Z}_{h}\right)}{N_{h}-1} S_{x z h}=\frac{\sum_{i=1}^{N_{h}}\left(\bar{x}_{h}-\bar{X}_{h}\right)\left(\bar{z}_{h}-\bar{Z}_{h}\right)}{N_{h}-1} \\
\gamma_{h}=\frac{1-f_{h}}{n_{h}} .
\end{gathered}
$$




\section{Adapted Estimators}

When the information on the two auxiliary variables is known, Singh $(1965,1967)$ proposed some ratio cum product estimators in simple random sampling to estimate the population mean of the study variable $y$.

Motivated by Singh $(1965,1967)$ and Singh, R. et. al. (2009), we propose some estimators in stratified sampling as

$$
\begin{aligned}
& \mathrm{t}_{1}=\overline{\mathrm{y}}_{\mathrm{st}} \exp \left[\begin{array}{l}
\overline{\mathrm{X}}-\overline{\mathrm{x}}_{\mathrm{st}} \\
\overline{\overline{\mathrm{X}}+\mathrm{x}_{\mathrm{st}}}
\end{array}\right] \exp \left[\begin{array}{l}
\overline{\bar{Z}}-\overline{\mathrm{z}}_{\mathrm{st}} \\
\overline{\overline{\mathrm{X}}+\overline{\mathrm{z}}_{\mathrm{st}}}
\end{array}\right] \\
& \mathrm{t}_{2}=\overline{\mathrm{y}}_{\mathrm{st}} \exp \left[\frac{\overline{\mathrm{x}}_{\mathrm{st}}-\overline{\mathrm{X}}}{\mathrm{x}_{\mathrm{st}}+\overline{\overline{\mathrm{X}}}}\right] \exp \left[\frac{\overline{\mathrm{z}}_{\mathrm{st}}-\overline{\mathrm{Z}}}{\overline{\mathrm{z}}_{\mathrm{st}}+\overline{\overline{\mathrm{Z}}}}\right] \\
& \mathrm{t}_{3}=\overline{\mathrm{y}}_{\mathrm{st}} \exp \left[\begin{array}{l}
\overline{\mathrm{X}}-\overline{\mathrm{x}}_{\mathrm{st}} \\
\overline{\overline{\mathrm{X}}}+\mathrm{x}_{\mathrm{st}}
\end{array}\right] \exp \left[\begin{array}{l}
\overline{\mathrm{z}}_{\mathrm{st}}-\overline{\mathrm{Z}} \\
\overline{\overline{\mathrm{z}}_{\mathrm{st}}+\overline{\overline{\mathrm{Z}}}}
\end{array}\right] \\
& \mathrm{t}_{4}=\overline{\mathrm{y}}_{\mathrm{st}} \exp \left[\frac{\overline{\mathrm{x}}_{\mathrm{st}}-\overline{\mathrm{X}}}{\mathrm{x}_{\mathrm{st}}+\overline{\overline{\mathrm{X}}}}\right] \exp \left[\frac{\overline{\overline{\mathrm{Z}}}-\overline{\mathrm{z}}_{\mathrm{st}}}{\overline{\overline{\mathrm{X}}}+\overline{\mathrm{z}}_{\mathrm{st}}}\right]
\end{aligned}
$$

The MSE equations of these estimators can be written as

$$
\begin{aligned}
& \operatorname{MSE}\left(\mathrm{t}_{1}\right)=\bar{Y}^{2}\left(V_{200}^{r}+\frac{V_{020}}{4}+\frac{V_{002}}{4}-V_{110}^{r}-V_{101}^{r}+\frac{V_{011}}{2}\right) \\
& \operatorname{MSE}\left(t_{2}\right)=\bar{Y}^{2}\left(V_{200}^{r}+\frac{V_{020}}{4}+\frac{V_{002}}{4}+V_{110}^{r}+V_{101}^{r}+\frac{V_{011}}{2}\right) \\
& \operatorname{MSE}\left(t_{3}\right)=\bar{Y}^{2}\left(V_{200}^{r}+\frac{V_{020}}{4}+\frac{V_{002}}{4}-V_{110}^{r}+V_{101}^{r}-\frac{V_{011}}{2}\right) \\
& \operatorname{MSE}\left(t_{4}\right)=\bar{Y}^{2}\left(V_{200}^{r}+\frac{V_{020}}{4}+\frac{V_{002}}{4}+V_{110}^{r}-V_{101}^{r}-\frac{V_{011}}{2}\right)
\end{aligned}
$$

\section{Suggested Estimators}

$$
t_{i}^{*}=\bar{y}_{s t} \exp \left|\frac{\left(A_{s t}^{*}+b_{s t}\right)-\left(a_{s t}^{*}+b_{s t}\right)}{\left(A_{s t}^{*}+b_{s t}\right)+\left(a_{s t}^{*}+b_{s t}\right)}\right|^{\alpha_{1}} \exp \left|\frac{\left(c_{s t}^{*}+d_{s t}\right)-\left(C_{s t}^{*}+d_{s t}\right)}{\left(c_{s t}^{*}+d_{s t}\right)+\left(C_{s t}^{*}+d_{s t}\right)}\right|^{\alpha_{2}}(i=1,2,3,4)
$$

Let $A_{s t}^{*}=\sum_{h=1}^{L} w_{h} \bar{X}_{h} A_{h}$ and $a_{s t}^{*}=\sum_{h=1}^{L} w_{h} \bar{x}_{h} A_{h}$, where $A_{h}$ may be some population information of the first auxiliary variable for $h^{\text {th }}$ stratum similarly, we define

$\mathrm{C}_{\mathrm{st}}^{*}=\sum_{\mathrm{h}=1}^{\mathrm{L}} \mathrm{w}_{\mathrm{h}} \overline{\mathrm{Z}}_{\mathrm{h}} \mathrm{B}_{\mathrm{h}}$ and $\mathrm{c}_{\mathrm{st}}^{*}=\sum_{\mathrm{h}=1}^{\mathrm{L}} \mathrm{w}_{\mathrm{h}} \overline{\mathrm{z}}_{\mathrm{h}} \mathrm{B}_{\mathrm{h}}$

where $B_{h}$ may be some population information of the second auxiliary variable for $h^{\text {th }}$ stratum.

Expressing $\mathrm{t}_{\mathrm{i}}^{*}$ in terms of e's, we have

$$
\mathrm{t}_{\mathrm{i}}^{*}=\overline{\mathrm{Y}}\left(1+\mathrm{e}_{0}\right) \exp \left|\frac{-\theta_{1}}{2} \mathrm{e}_{1}^{*}\left(1+\frac{\theta_{1}}{2} \mathrm{e}_{1}^{*}\right)^{-1}\right|^{\alpha_{1}} \exp \left|\frac{\theta_{2}}{2} \mathrm{e}_{2}^{*}\left(1+\frac{\theta_{2}}{2} \mathrm{e}_{2}^{*}\right)^{-1}\right|^{\alpha_{2}}
$$


where,

$$
\begin{aligned}
& \theta_{1}=\frac{A_{\mathrm{st}}^{*}}{\mathrm{~A}_{\mathrm{st}}^{*}+\mathrm{b}_{\mathrm{st}}}, \quad \theta_{2}=\frac{\mathrm{C}_{\mathrm{st}}^{*}}{\mathrm{C}_{\mathrm{st}}^{*}+\mathrm{d}_{\mathrm{st}}} \\
& \mathrm{e}_{1}^{*}=\frac{\mathrm{a}_{\mathrm{st}}^{*}-\mathrm{A}_{\mathrm{st}}^{*}}{\mathrm{~A}_{\mathrm{st}}^{*}}=\frac{\sum_{\mathrm{h}=1}^{\mathrm{L}} \mathrm{w}_{\mathrm{h}} \mathrm{A}_{\mathrm{h}}\left(\overline{\mathrm{x}}_{\mathrm{h}}-\overline{\mathrm{X}}_{\mathrm{h}}\right)}{\mathrm{A}_{\mathrm{st}}^{*}}
\end{aligned}
$$

and

$\mathrm{e}_{2}^{*}=\frac{\mathrm{c}_{\mathrm{st}}^{*}-\mathrm{C}_{\mathrm{st}}^{*}}{\mathrm{C}_{\mathrm{st}}^{*}}=\frac{\sum_{\mathrm{h}=1}^{\mathrm{L}} \mathrm{w}_{\mathrm{h}} \mathrm{B}_{\mathrm{h}}\left(\overline{\mathrm{x}}_{\mathrm{h}}-\overline{\mathrm{X}}_{\mathrm{h}}\right)}{\mathrm{C}_{\mathrm{st}}^{*}}$

It is obvious that $\mathrm{E}\left(\mathrm{e}_{1}^{*}\right)=\mathrm{E}\left(\mathrm{e}_{2}^{*}\right)=0$.

Expending the right hand side of (2.10) and retaining the terms up to first order of approximation, we get

$$
\begin{aligned}
\left(\mathrm{t}_{\mathrm{i}}^{*}-\overline{\mathrm{Y}}\right) \cong \overline{\mathrm{Y}}\left[\mathrm{e}_{0}-\frac{1}{2}\left(\alpha_{1} \theta_{1} \mathrm{e}_{1}^{*}-\right.\right. & \left.\alpha_{2} \theta_{2} \mathrm{e}_{2}^{*}\right)+\frac{1}{4}\left(\alpha_{1} \theta_{1}^{2} \mathrm{e}_{1}^{* 2}-\alpha_{2} \theta_{2}^{2} \mathrm{e}_{2}^{* 2}\right) \\
& +\alpha_{1}\left(\alpha_{1}-1\right) \frac{\theta_{1}^{2} e_{1}^{* 2}}{4}+\alpha_{2}\left(\alpha_{2}-1\right) \frac{\theta_{2}^{* 2} \mathrm{e}_{2}^{* 2}}{4}
\end{aligned}
$$

Squaring (3.3) to the first order of approximation and then taking expectation, we have -

$$
\begin{array}{r}
\operatorname{MSE}\left(\mathrm{t}_{\mathrm{i}}^{*}\right) \cong \overline{\mathrm{Y}}^{2}\left[\bigvee_{200}^{*}+\frac{1}{4}\left\{\alpha_{1}^{2} \theta_{1}^{* 2} \bigvee_{020}^{*}+\alpha_{2}^{2} \theta_{2}^{* 2} \bigvee_{002}^{*}\right\}\right. \\
\left.-\alpha_{1} \theta_{1}^{*} \bigvee_{110}^{*}+\alpha_{2} \theta_{2}^{*} \bigvee_{101}^{*}-\frac{1}{2} \alpha_{1} \alpha_{2} \theta_{1}^{*} \theta_{2}^{*} \bigvee_{011}^{*}\right]
\end{array}
$$

where,

$$
\begin{aligned}
& \theta_{1}^{*}=\frac{\overline{\mathrm{X}}_{\mathrm{st}}}{\mathrm{A}_{\mathrm{st}}^{*}+\mathrm{b}_{\mathrm{st}}}, \quad \theta_{2}^{*}=\frac{\overline{\mathrm{Z}}_{\mathrm{st}}}{\mathrm{C}_{\mathrm{st}}^{*}+\mathrm{d}_{\mathrm{st}}}, \\
& V_{\mathrm{rst}}^{*}=>_{\mathrm{i}=1}^{\mathrm{L}} \mathrm{W}^{\mathrm{r}+\mathrm{s}+\mathrm{t}}\left(\mathrm{A}_{\mathrm{h}}\right)^{\mathrm{s}}\left(\mathrm{B}_{\mathrm{h}}\right)^{\mathrm{t}} \frac{\mathrm{E}\left[\left(\overline{\mathrm{y}}_{\mathrm{h}}-\overline{\mathrm{Y}}_{\mathrm{h}}\right)^{\mathrm{r}}\left(\overline{\mathrm{x}}_{\mathrm{h}}-\overline{\mathrm{X}}_{\mathrm{h}}\right)^{\mathrm{s}}\left(\overline{\mathrm{z}}_{\mathrm{h}}-\overline{\mathrm{Z}}_{\mathrm{h}}\right)^{\mathrm{t}}\right]}{\overline{\mathrm{Y}}^{\mathrm{r}} \overline{\mathrm{X}}^{\mathrm{s}} \overline{\mathrm{Z}}^{\mathrm{t}}} .
\end{aligned}
$$

On differentiating (3.4) with respect to $\alpha_{1}$ and $\alpha_{2}$, respectively we get the optimum values of $\alpha_{1}$ and $\alpha_{2}$ as:

$$
\alpha_{1}^{*}=\frac{2\left(V_{002}^{*} V_{110}^{*}-V_{101}^{*} V_{011}^{*}\right)}{\theta_{1}^{*}\left(V_{020}^{*} V_{002}^{*}-V_{011}^{*}{ }^{2}\right)}
$$

and

$$
\alpha_{2}^{*}=\frac{2\left(\mathrm{~V}_{011}^{*} \mathrm{~V}_{110}^{*}-\mathrm{V}_{101}^{*} \mathrm{~V}_{020}^{*}\right)}{\theta_{2}^{*}\left(\mathrm{~V}_{020}^{*} \mathrm{~V}_{002}^{*}-\mathrm{V}_{011}^{*}{ }^{2}\right)}
$$


Putting optimum values of $\alpha_{1}^{*}$ and $\alpha_{2}^{*}$ from (3.5) and (3.6) in (3.4) we obtain min. MSE of $\mathrm{t}_{\mathrm{i}}^{*}$ as-

$\operatorname{MSE}\left(\mathrm{t}_{\mathrm{i}}^{*}\right) \min =\overline{\mathrm{Y}}^{2}\left(\mathrm{~V}_{200}^{*}-\frac{\mathrm{V}_{002}^{*} \mathrm{~V}_{110}^{* 2}+\mathrm{V}_{020}^{*} \mathrm{~V}_{110}^{* 2}-2 \mathrm{~V}_{110}^{r} \mathrm{~V}_{101} \mathrm{~V}_{011}}{\mathrm{~V}_{020}^{*} \mathrm{~V}_{002}^{*}-\mathrm{V}_{011}^{* 2}}\right)$

\section{Efficiency comparison}

In this section, firstly, we compare the MSE of proposed estimator $t_{i}^{*}(i=1,2,3,4)$ with $\mathrm{V}\left(\overline{\mathrm{y}}_{\mathrm{st}}\right)$.

$$
\begin{gathered}
\operatorname{MSE}\left(\mathrm{t}_{\mathrm{i}}^{*}\right)<V\left(\overline{\mathrm{y}}_{\mathrm{st}}\right) \\
\overline{\mathrm{Y}}^{2}\left|\mathrm{~V}_{200}^{*}+\frac{1}{4}\left\{\alpha_{1}^{2} \theta_{1}^{* 2} \bigvee_{020}^{*}+\alpha_{2}^{2} \theta_{2}^{* 2} \bigvee_{002}^{*}\right\}-\alpha_{1} \theta_{1}^{*} \bigvee_{110}^{*}+\alpha_{2} \theta_{2}^{*} V_{101}^{*}-\frac{1}{2} \alpha_{1} \alpha_{2} \theta_{1}^{*} \theta_{2}^{*} V_{011}^{*}\right| \\
<\overline{\mathrm{Y}}^{2} V_{200}^{*}
\end{gathered}
$$

Estimator $\mathrm{t}_{\mathrm{i}}^{*}(\mathrm{i}=1,2,3,4)$ performs better than $\overline{\mathrm{y}}_{\mathrm{st}}$, whenever

$$
\frac{2 A}{B}>1
$$

where, $A=\alpha_{1} \alpha_{2} \theta_{1}^{*} \theta_{2}^{*} V_{011}^{*}+2 \alpha_{1} \theta_{1}^{*} V_{110}^{*}-2 \alpha_{2} \theta_{2}^{*} V_{101}^{*}$

$$
\mathrm{B}=\alpha_{1}^{2} \theta_{1}^{* 2} V_{020}^{*}+\alpha_{2}^{2} \theta_{2}^{* 2} V_{002}^{*}
$$

Secondly, we find the condition under which $\operatorname{MSE}\left(\mathrm{t}_{\mathrm{i}}^{*}\right)$ min performs better than $\mathrm{V}\left(\mathrm{y}_{\mathrm{st}}\right)$ $\operatorname{MSE}\left(\mathrm{t}_{\mathrm{i}}^{*}\right) \min <\mathrm{V}\left(\overline{\mathrm{y}}_{\mathrm{st}}\right)$

$\overline{\mathrm{Y}}^{2}\left(\mathrm{~V}_{200}^{*}-\frac{\mathrm{V}_{002}^{*} \mathrm{~V}_{110}^{* 2}+\mathrm{V}_{020}^{*} \mathrm{~V}_{110}^{* 2}-2 \mathrm{~V}_{110}^{r} \mathrm{~V}_{101}^{r} \mathrm{~V}_{011}^{r}}{\mathrm{~V}_{020}^{*} \mathrm{~V}_{002}^{*}-\mathrm{V}_{011}^{* 2}}\right)<\overline{\mathrm{Y}}^{2} \mathrm{~V}_{200}^{*}$

$\operatorname{MSE}\left(\mathrm{t}_{\mathrm{i}}^{*}\right) \min$ is less than $\mathrm{V}\left(\mathrm{y}_{\mathrm{st}}\right)$, whenever

$\frac{\mathrm{C}}{\mathrm{D}}>0$

where,

$$
\begin{aligned}
& C=V_{002}^{*} V_{110}^{* 2}+V_{020}^{*} V_{110}^{* 2}-2 V_{110}^{r} V_{101}^{r} V_{011}^{r} \\
& \quad \text { and } \quad D=V_{020}^{*} V_{002}^{*}-V_{011}^{* 2} .
\end{aligned}
$$

\section{Empirical study}

For empirical study we use the data set which is earlier used by Koyuncu and Kadilar (2009). 


\section{Data Statistics}

\begin{tabular}{|c|c|c|}
\hline$N_{1}=127$ & $\mathrm{~N}_{2}=117$ & $N_{3}=103$ \\
\hline$N_{4}=170$ & $N_{5}=205$ & $N_{6}=201$ \\
\hline $\mathrm{n}_{1}=31$ & $\mathrm{n}_{2}=21$ & $\mathrm{n}_{3}=29$ \\
\hline $\mathrm{n}_{4}=38$ & $\mathrm{n}_{5}=22$ & $\mathrm{n}_{6}=39$ \\
\hline$S_{y 1}=883.835$ & $S_{y 2}=644$ & $S_{y 3}=1033.467$ \\
\hline$S_{y 4}=810.585$ & $S_{y 5}=403.654$ & $S_{y 6}=711.723$ \\
\hline$\overline{\mathrm{Y}}_{1}=703.74$ & $\bar{Y}_{2}=413$ & $\overline{\mathrm{Y}}_{3}=573.17$ \\
\hline $\bar{Y}_{4}=424.66$ & $\bar{Y}_{5}=267.03$ & $\bar{Y}_{6}=393.84$ \\
\hline$S_{x 1}=30486.751$ & $S_{x 2}=15180.760$ & $S_{x 3}=27549.697$ \\
\hline$S_{x 4}=18218.931$ & $S_{x 5}=8997.776$ & $S_{x 6}=23094.141$ \\
\hline$\overline{\mathrm{X}}_{1}=20804.59$ & $\bar{X}_{2}=9211.79$ & $\bar{X}_{3}=14309.30$ \\
\hline $\bar{X}_{4}=9478.85$ & $\bar{X}_{5}=5569.95$ & $\bar{X}_{6}=12997.59$ \\
\hline$S_{x y 1}=25237153.52$ & $S_{x y 2}=9747942.85$ & $S_{x y 3}=28294397.04$ \\
\hline$S_{x y 1}=14523885.53$ & $S_{x y 1}=3393591.75$ & $S_{x y 6}=15864573.97$ \\
\hline$\rho_{x y 1}=0.936$ & $\rho_{x y 2}=0.996$ & $\rho_{\mathrm{xy} 3}=0.994$ \\
\hline$\rho_{x y 4}=0.983$ & $\rho_{x y 5}=0.989$ & $\rho_{\mathrm{xy} 6}=0.965$ \\
\hline$\beta_{2}\left(x_{1}\right)=4.593$ & $\beta_{2}\left(x_{2}\right)=18.543$ & $\beta_{2}\left(x_{3}\right)=15.446$ \\
\hline$\beta_{2}\left(x_{4}\right)=10.162$ & $\beta_{2}\left(x_{5}\right)=21.947$ & $\beta_{2}\left(x_{6}\right)=23.114$ \\
\hline$\beta_{2}\left(y_{1}\right)=2.158$ & $\beta_{2}\left(y_{2}\right)=16.392$ & $\beta_{2}\left(y_{3}\right)=14.979$ \\
\hline$\beta_{2}\left(y_{4}\right)=12.167$ & $\beta_{2}\left(y_{5}\right)=21.088$ & $\beta_{2}\left(y_{6}\right)=20.254$ \\
\hline$S_{Z 1}=555.5816$ & $S_{22}=365.4576$ & $S_{z 3}=612.9509281$ \\
\hline$S_{Z 4}=458.0282$ & $S_{25}=260.8511$ & $S_{\mathrm{z}}=397.0481$ \\
\hline$\overline{\mathrm{Z}}_{1}=498.28$ & $\overline{\mathrm{Z}}_{2}=318.33$ & $\overline{\mathrm{Z}}_{3}=431.36$ \\
\hline$\overline{\mathrm{Z}}_{4}=498.28$ & $\overline{\mathrm{Z}}_{5}=227.20$ & $\overline{\mathrm{Z}}_{6}=313.71$ \\
\hline$S_{y z 1}=480688.2$ & $S_{y z 2}=230092.8$ & $S_{y z 1}=623019.3$ \\
\hline$S_{y z 1}=364943.4$ & $S_{y z 1}=101539$ & $S_{y z 1}=277696.1$ \\
\hline$S_{\mathrm{xz} 1}=15914648$ & $S_{x z 2}=5379190$ & $S_{x z 3}=164900674.56$ \\
\hline$S_{x z 4}=8041254$ & $S_{\mathrm{xz} 5}=2144057$ & $S_{\mathrm{xz1} 1}=8857729$ \\
\hline$\rho_{y z 1}=0.978914$ & $\rho_{y z 2}=0.9762$ & $\rho_{y 3}=0.983511$ \\
\hline$\rho_{y z 4}=0.982958$ & $\rho_{y z 5}=0.964342$ & $\rho_{y z 1}=0.982689$ \\
\hline$\beta_{2}\left(z_{1}\right)=2.314926$ & $\beta_{2}\left(z_{2}\right)=11.19093$ & $\beta_{2}\left(z_{3}\right)=10.78635$ \\
\hline$\beta_{2}\left(\mathrm{z}_{4}\right)=8.624111$ & $\beta_{2}\left(z_{5}\right)=9.720886$ & $\beta_{2}\left(z_{6}\right)=14.40696$ \\
\hline
\end{tabular}


Table1: Percent Relative Efficiencies (PRE) of estimators

\begin{tabular}{|c|c|c|c|c|c|}
\hline Estimator & $\boldsymbol{\alpha}_{\mathbf{1}}$ & $\boldsymbol{\alpha}_{\mathbf{2}}$ & $\mathbf{A}_{\mathbf{h}}$ & $\mathbf{B}_{\mathbf{h}}$ & PRE \\
\hline$\overline{\mathrm{y}}_{\mathrm{st}}$ & 0 & 0 & 1 & 1 & 100 \\
\hline $\mathrm{t}_{1}$ & 1 & -1 & 1 & 1 & 2045.439 \\
\hline $\mathrm{t}_{2}$ & -1 & 1 & 1 & 1 & 27.948 \\
\hline $\mathrm{t}_{3}$ & 1 & 1 & 1 & 1 & 126.419 \\
\hline $\mathrm{t}_{4}$ & -1 & -1 & 1 & 1 & 77.219 \\
\hline $\mathrm{t}_{1}^{*}$ & 0.0215 & 0.140416 & $\beta_{2}(\mathrm{x})$ & $\beta_{2}(\mathrm{z})$ & 101.155 \\
\hline $\mathrm{t}_{2}^{*}$ & 0.6893 & -0.9946 & $\mathrm{C}_{\mathrm{xh}}$ & $\mathrm{C}_{\mathrm{zh}}$ & 256.181 \\
\hline $\mathrm{t}_{3}^{*}$ & 0.5835 & -1.7739 & 1 & 1 & 2854.597 \\
\hline $\mathrm{t}_{4}^{*}$ & 0.7533 & -1.5332 & $\rho_{\mathrm{xy}}$ & $\rho_{\mathrm{yz}}$ & 7251.178 \\
\hline
\end{tabular}

\section{Conclusion}

From Table 1, we observe that the estimator $t_{4}^{*}$ which uses correlation coefficient as $A_{h}$ and $B_{h}$ has the smallest MSE value among their own family of estimators. When we further examine Table 1, we see that all exponential ratiotype estimators performs better than usual mean estimator $\bar{y}_{\text {st }}$.

\section{References}

1. Bahl. S. and Tuteja, R.K. (1991): Ratio and Product Type Exponential Estimator. Infrm. and Optim. Sci., XIII, 159-163.

2. Diana, G. (1993): A class of estimators of the population mean in stratified random sampling. Statistica 53(1), 59-66.

3. Ghosh, S.P. (1958). A Note on stratified random sampling with multiple characteristics. Calc. Stat. Assoc. Bull., 8, (30-31), 81-90.

4. Kadilar, C., Cingi, H. (2003): Ratio estimators in stratified random sampling. Biometr. J.45, 218-225.

5. Koyuncu, N. and Kadilar, C. (2009): Family of Estimators of Population Mean Using Two Auxiliary Variables in Stratified Random Sampling. Commun.in Statist. Theo. and Meth., 38: 2398-2417.

6. Perri, P. F. (2007): Improved ratio-cum-product type estimators. Statist. Trans. 8:51-69.

7. Rao, T.J. (1977). Optimum allocation of sample size and prior distributions: A review. Intern. Stat. Rev., 45, 173-179.

8. Singh, H. P., Vishwakarma, G. K. (2008): A family of estimators of population mean using auxiliary information in stratified sampling. Commun. Statist. Theo. Meth.37, 1038-1050. 
9. Singh, H. P., Tailor, R., Singh, S., Kim, J. M. (2008): A modified estimator of population mean using power transformation. Statist.Pap.49:37-58.

10. Singh, M. P. (1965): On the estimation of ratio and product of the population parameters. Sankhya B 27:231-328.

11. Singh, M. P. (1967). Ratio cum product method of estimation. Metrika 12: 34-42.

12. Singh, R., Cauhan, P., Sawan, N. and Smarandache, F. (2007): Auxiliary information and a priori values in construction of improved estimators. Renaissance High press.

13. Singh, R., Kumar, M., Singh, R.D., and Chaudhary, M.K. (2008): Exponential Ratio Type Estimators in Stratified Random Sampling. (Proceedings of International Symposium on Optimisation and Statistics (I.S.O.S.), 2008 held at A.M.U., Aligarh, India, during 29-31 Dec 2008).

14. Singh, R., Chauhan, P., Sawan, N. and Smarandache, F. (2009): Improvement in estimating the population mean using exponential estimator in simple random sampling. Bull. of Stat. and Econ., 3, 13-18. 